\title{
6 Schlüsselakteure der Regionalentwicklung: Die Sicht innovativer Unternehmen auf die Politik
}

Innovative Unternehmen spielen eine wichtige Rolle bei der Stärkung ländlicher Regionen. Eine Diversifizierung der regionalen Wirtschaft und attraktive Arbeitsplätze in zukunftsträchtigen Branchen gelten als wichtiger Faktor, um diese Gebiete auch für junge Menschen attraktiver zu machen. Folglich ist auch die Politik bemüht, diese Unternehmen anzusiedeln beziehungsweise ihre Gründung in ländlichen Regionen zu ermöglichen. Dieser Abschnitt geht folglich darauf ein, wie die befragten Unternehmen das Zusammenspiel mit der Politik bewerten und wo noch Handlungsbedarf gesehen wird. Einige Punkte wurden bereits in Kapitel 5.2.2 unter dem Stichwort „Gestaltungsspielraum“" angesprochen. Neben der Lokalpolitik spielen aber natürlich auch die Strategien von Bund und Ländern eine Rolle. Im Großen und Ganzen zeigen sich die befragten Unternehmen zufrieden mit ihrem Verhältnis zu den politischen Akteurinnen und Akteuren, wobei die Beziehung sehr unterschiedlich gestaltet ist. Die meisten sprechen von professioneller Distanz, das heißt, man kennt sich und kommt gut miteinander aus, versucht aber auf einer professionellen und nicht einer informellen Ebene zusammenzuarbeiten. Oft wird auch betont, dass das in der Vergangenheit anders war, dass mittlerweile aber juristisch eindeutige Beschlüsse oberste Priorität haben. Diese Professionalisierung wird durchwegs positiv gesehen:

„Aber ich glaube, man muss sich der Sache schon bewusst sein als solches. Auch wir
möchten Rechtssicherheit haben und ich möchte keine rechtliche Grauzone haben, son-
dern das sollte alles mit Fug und Recht auch genehmigt sein.“ [Unternehmen M]

„Wir brauchen weniger Kontakte jetzt, die helfen dir jetzt nicht, dass du in Österreich irgendein Projekt gewinnst, die Zeiten sind vorbei.“ [Unternehmen J]

„Natürlich, wenn man jetzt den Bürgermeister an der Bar trifft, dann trinkt man auch einmal ein Bier, aber prinzipiell schon professionelle Distanz. Kann er sich nicht leisten und kommt auch, naja, macht auch keinen Sinn. Ich bin immer dafür, mit Profis zu arbeiten, das ist viel, viel einfacher.“ [Unternehmen Q]

„Ich würde einmal sagen, die lokale Behörde, mit der haben wir eine sehr gute Zusammenarbeit. Auch mit dem Land gibt es eine gute Zusammenarbeit auf einer sehr sachlichen, fairen Ebene. Aber ich habe auch nicht das Gefühl, dass wir jetzt bevorzugt behandelt werden. “ [Unternehmen A]

„Hier gibt es auch nichts mehr mit Sondergenehmigungen und Augenzwinkern oder irgend so etwas, lange gibt es das nicht mehr. Und hier muss ich ehrlich sagen, hier ist das 
Land und auch hier die Region immer sehr kooperativ und vor allem lösungsorientiert.“ [Unternehmen C]

Die Art der Kontakte ist dabei sehr unterschiedlich. Einige Unternehmen arbeiten hier sehr eigenständig und sehen für die Politik auch nicht wirklich einen Spielraum, unterstützend tätig zu werden. Andere sind proaktiv und in diversen Gremien aktiv, um den Austausch mit der Politik zu intensivieren und um Verbesserungen zu erreichen. Wieder andere Unternehmen suchen derzeit das Gespräch, um nicht nur auf lokaler oder regionaler Ebene, sondern auch hinsichtlich der Gesetzgebung auf Bundesebene Verbesserungen zu erreichen, auch wenn es vor allem für junge Unternehmen schwierig ist, die richtigen Ansprechpersonen zu finden:

„Wir haben keinen Kontakt mit der Politik. Also schon Kontakt mit der Politik, aber wir versuchen schon einigermaßen gut auszukommen mit ihnen, aber an und für sich haben wir keinen Kontakt.“ [Unternehmen N]

„Was einfach ganz wichtig ist für junge und innovative Betriebe, vor allem in der Frühphase: Es fehlt oft der Zugang zu bestimmten Ansprechpartnern. Das ist bei uns immer noch so, wir suchen für ganz spezielle Teilbereiche (...) immer wieder Experten, wo wir uns sehr schwer tun, jemanden zu finden. Auch nicht für Geld. Oder wir finden gar keinen, weil wir nicht wissen, wen fragen." [Unternehmen $\mathrm{H}]$

„Ich wünschte mir mehr Kontakt, es gibt einige Punkte, die mir wirklich weh tun. Wo ich mir denke, wenn man das ändert, könnte man ein paar Startups länger leben lassen oder zumindest in die Gewinnschwelle bringen. (...) Gerade im digitalen Wandel gibt es einige Sachen, die man in der Gesetzgebung diskutieren müsste.“ [Unternehmen B]

Ganz generell werden die öffentliche Förderlandschaft und die Politik meistens positiv gesehen. Einige Firmen sind in Clusternetzwerken aktiv und sind mit dem Clustermanagement meistens zufrieden. Das zeigt, das Cluster nach wie vor oft geographisch gedacht werden, sich Unternehmen aber mittlerweile vor allem aus fachlichen und nicht räumlichen Gründen vernetzen. Weiters werden die Außenhandelsstellen der Wirtschaftskammer (WKO) genutzt und auch die Wichtigkeit der Forschungsförderungsgesellschaft (FFG) wird häufig hervorgehoben. Manche Programme erfordern aber mittlerweile ein sehr hohes Maß an Bürokratie, die in der Entwicklung hemmend wirkt:

„Also in der Entwicklung ist das extrem hemmend, weil ein Entwickler ist grundsätzlich einer (...), der ja auch ein bisschen einen anderen Zugang zur Materie hat. Ein Entwickler, der innovativ ist, ist nie ein Bürokrat.“ [Unternehmen S]

„Österreich ist ein Land, das über Netzwerke sich auch sehr gut entwickelt hat. Und das ist auch das, was Österreich ausmacht. (...) Wenn man weiß, wie gut die Außenhandels- 
stellen oder die Außenhandelsorganisation des Außenministeriums oder der Wirtschaftskammer funktionieren, das ist gigantisch eigentlich.“ [Unternehmen K]

„Das war ein klassisches FFG-Projekt im Sinne der Zusammenarbeit von Unternehmen und Universität, durch diese Mittel wurde das Projekt erst möglich. Ich würde sagen, FFG-Projekte an Standorten im ländlichen Raum sind besonders effektiv. Ist es erfolgreich, gibt es unmittelbar einen Wertschöpfungseffekt in der Region. Wir haben dadurch die Entwicklungsabteilung gegründet und Stellen in der Produktion geschaffen - und den Standort durch das neue, einzigartige Knowhow abgesichert.“ [Unternehmen G]

Oftmals wird die Zusammenarbeit auch durch die fehlende Kontinuität erschwert, wenn im Zuge von Wahlen die politischen Personen wechseln, wobei sich dies positiv sowie negativ auswirken kann. Dies unterstreicht auch die Bedeutung von Individuen, denen in der Regionalentwicklung oft eine entscheidende Rolle zukommt und die ein besonderes Verständnis für die örtlichen Fragestellungen haben (GAILING und IBERT 2016):

„Wenn der Landeshauptmann wechselt, wechselt auch meistens die Garnitur dahinter. Aber die dritte Garnitur bleibt und mit denen arbeite ich zusammen.“ [Unternehmen O]

„Was ich sagen muss, ist, dass es sich hier stark verändert hat, zum Positiven. Durch politische Wahlen ist halt der richtige Bürgermeister jetzt am richtigen Stuhl. Und da ist es schon auch ganz extrem toll, auf Augenhöhe mit der Politik reden zu können.“

[Unternehmen Q]

„Die Gemeinde ist sehr umtriebig, der Bürgermeister ist extrem gut vernetzt und dem liegt die Wirtschaft am Herzen. Wenn die Gemeinde etwas investiert, dann schaut er immer darauf, dass es lokale Firmen bekommen, dass die Wertschöpfung hierbleibt.“ [Unternehmen $\mathrm{H}$ ]

„Zu Beginn wären Förderungen wichtig gewesen, wir bekamen sie leider nicht. Es wurde befunden, dass es das schon gibt, was wir vorhaben. Das ist ein großer Kritikpunkt, dass irgendwer aus der Ferne bestimmt, der wohl auch nicht im Thema ist, ob du förderungswürdig bist oder nicht. (...) Die Gemeinde war gerade zum Start des Unternehmens eine starke Stütze, auch die Bank im Ort, die damals dann den Kredit gewährte.“

[Unternehmen L]

„Die Gemeinde denkt hier nicht sehr unternehmerisch. Sie möchte es, aber sie kann es nicht. (...) Also, man macht gern einen Handschlag und sagt, wir schauen, aber das ist eine Mindestvoraussetzung, weil ja Kommunalsteuern gezahlt und Mitarbeiter hier beschäftig werden." [Unternehmen K] 
Manchmal fehlt auch das Verständnis für neue, innovative Branchen in peripheren Regionen, aber auch bei Förderinstitutionen generell. Vor allem junge Unternehmen beschreiben, dass es für sie anfangs schwierig ist, mit ihren Anliegen durchzudringen und in der Region bekannt zu werden. Innovative Unternehmen sind vor Ort natürlich gerne gesehen, aber beim Bewusstsein für konkrete Unterstützungsmaßnahmen stellt sich der Sachverhalt komplizierter dar:

„Das Zugpferd ist der Tourismus. Das Zugpferd ist die Gastronomie. Und es ist der Gast, der das Geld dalässt. (...) Das heißt, es lebt eigentlich jeder von dieser Branche. Wir leben unmittelbar eigentlich von dem gar nicht. (...) Und darum, glaube ich, hat der Bürgermeister, wenn man so sagt, in seinem Wirken, sicher sein Denken ganz woanders hin ausgerichtet.“ [Unternehmen I]

„Sie schreiben sich das alle auf die Brust und sagen, die Unternehmen sind super und wichtig, aber im Endeffekt, dass sie uns irgendwie unterstützen, das würde ich eher so sagen, ist auf einem geringen Niveau.“ [Unternehmen P]

„Wir wissen, dass wir ein enormes Wachstumspotential haben. Das ist ein Potential, dass es in der klassischen Wirtschaftswelt sonst nicht gibt, weil es einfach ein so ein potentes, digitales Produkt ist. Das ist etwas, wo die Gemeinde mit Sicherheit partizipiert, aber ich glaube, dass sich die Gemeinde auch generell dann so darstellen kann, als eine Gemeinde oder ein Ort, der am Puls der Zeit ist." [Unternehmen B]

Doch auch die supranationale Ebene (beispielsweise EU-Richtlinien) hat für einige zukunftsträchtige Branchen eine wichtige Bedeutung, wenn Rahmenbedingungen gesetzt werden, die für die eigenen Produkte große Auswirkungen haben. Hier lässt sich schwer planen, ob Förderungen tatsächlich wie angekündigt zur Verfügung stehen. Für eine Pfadentwicklung sind also nicht nur historische Ereignisse entscheidend, sondern auch zukünftige Ziele und die entsprechenden politischen Rahmenbedingungen, um diese zu erreichen (STEEN und HANSEN 2018):

„Der Markt ist natürlich sehr stark politisch beeinflusst und beeinflussbar. Es gibt einen Plan der EU mit der EU-Richtlinie, welche Ausbauzahlen für 2020 und 2025 erreicht werden sollen. (...) Da zeigt sich, dass wir fast $50 \%$ unter der Erwartungshaltung liegen. Was natürlich beeinträchtigend ist, für unsere Budgetierung und Planung mit Wachstum und Co, weil wenn diese Zahlen am Markt nicht erreicht werden, dann werden wir auch unsere Umsätze nicht erreichen. (...) Man kann es nicht beeinflussen, ob jetzt eine Förderung zusätzlich auf einmal ausgesprochen wird, oder vielleicht eine in eine Richtung ausgegeben wird, die man anders eingeschätzt hätte.“ [Unternehmen I]

„Also, wenn man es auf die Politik bezieht, es gibt keine klaren Regeln. Wenn irgendein Land sagt, ab 2030 ist diese Technologie verboten. So. Aber keiner definiert, was ist bis 
2030. Oder 2020. Egal. Dann ist das Ergebnis, bis 2020 wird die alte Technologie eingebaut, weil es nachher nicht erlaubt ist.“ [Unternehmen S]

Schließlich ist die Politik natürlich nicht nur auf der unternehmerischen Ebene gefordert, es ist auch ein ganzheitlicher Blick auf die Region notwendig. Einige Unternehmen sind sich durchaus bewusst, dass in ihrer Region der demographische Wandel besonders viele Herausforderungen mit sich bringen wird. Einige Großunternehmen engagieren sich auch aktiv, um die regionalen Rahmenbedingen zu verbessern, etwa indem man sich um die Errichtung einer tertiären Bildungseinrichtung vor Ort bemüht. Generell wird die Gestaltung dieser Rahmenbedingungen aber als Aufgabengebiet der Politik gesehen. Diese ist hier gefordert Maßnahmen zu ergreifen, damit es auch in Zukunft in ländlichen Regionen möglich ist, ein Unternehmen erfolgreich zu führen. Dabei äußern manche befragte Unternehmen auch Verständnis, dass es sich hier um keine triviale Herausforderung handelt:

„Das Thema Brain-Drain beschäftigt uns natürlich. Ich meine, wenn man so die Bevölkerungsentwicklung der Bezirke anschaut, sieht es hier ja so aus, dass wir uns hier verschlanken, in Anführungszeichen. (...) Ich glaube auch nicht, dass alle in der Stadt leben müssen und können. Und hier braucht es schon auch Anstrengungen im peripheren Raum, dass hier etwas weitergeht." [Unternehmen F]

„Nachdem hier geschichtlich gesehen wenig Bedarf ist, war auch niemals der Drang da, von qualifizierten Fachkräften jetzt großartig hierher einzureisen beziehungsweise für Unis, hier ihren Standort aufzubauen, weil sie halt keiner braucht. Und natürlich ist es so, dass die Firmen, die jetzt aktuell hier sind, dementsprechend auch (...) Fachkräftemangel haben. (...) Aber ja, so über einen Kamm zu scheren und zu sagen, es sind keine Unis da und deswegen haben wir keine Innovationskraft hier, das ist ein Henne-Ei-Problem.“ [Unternehmen D]

„Die Landflucht von jungen Technikern stellt für uns allerdings langfristig ein großes Problem dar. Ich bin mir bewusst, dass es eine enorme Herausforderung für die Politik ist, diesen aufzuhalten, dennoch muss alles versucht werden, hier gegenzusteuern, um ländliche Raume zu stabilisieren.“ [Unternehmen G]

Ein erster Schritt könnte eine intensive Auseinandersetzung mit dem Image ländlicher Regionen sein. Oftmals überwiegen in der öffentlichen Wahrnehmung negative Assoziationen, die ein Mitgrund sein können, dass sich hochqualifizierte Arbeitskräfte nicht auf Stellen in diesen Gebieten bewerben, auch wenn sie die Lebensqualität dort grundsätzlich schätzen. Ein Ziel könnte es folglich sein, auch andere innovative Branchen neben Landwirtschaft und Tourismus im ländlichen Raum hervorzuheben und auch in der öffentlichen Wahrnehmung stärker zu etablieren. Man könnte also ein positives Framing für den ländlichen Raum entwickeln (HEINTEL 2019), das über landschaftliche 
Schönheit hinausgeht, um langfristig einen Imagewandel zu erreichen. Ein Unternehmen sieht diesbezüglich einen besonders großen Handlungsbedarf:

„Ich möchte meinen Mitarbeitern nicht sagen, sie sollen in den ländlichen Raum gehen, sondern sie sollen in eine innovative Region kommen. (...) Man hat ja diese Cluster-Bildung (...) gemacht, gibt es ja in den ganzen Regionen. Die haben jetzt alle Firmen, aber was sie nicht gemacht haben, war die Aufwertung der Region. Und zwar auch die Wahrnehmung im Kopf drinnen. Der Begriff „ländlicher Raum“ ist nur gut für den Tourismus. Und wenn wir unsere Umsatzzahlen in Österreich anschauen, wir leben nicht nur vom Tourismus. Und da muss man etwas ändern, das ist die Aufgabe der Politik. Ich unterstütze sie jederzeit, aber da müssten wir etwas ändern daran.“ [Unternehmen Q]

Die Zusammenarbeit mit der Politik und öffentlichen Einrichtungen wird von den befragten Unternehmen demnach sehr unterschiedlich bewertet und gehandhabt. Während einige in diversen Gremien wie Forschungsbeiräten aktiv sind und auch informelle Kontakte pflegen, arbeiten andere nur, wenn es notwendig ist, mit der Politik zusammen. Das kann an der Unternehmenskultur liegen, wenn sie darauf ausgerichtet ist, möglichst wenig an der Politik anzustreifen. Oder auch daran, ob ein gutes Verhältnis zu den handelnden Akteurinnen und Akteuren besteht. Einige Unternehmen beschreiben, dass sich die Kooperation verbessert oder verschlechtert hat, nachdem in Folge von Wahlen auch die wesentlichen politischen Personen andere waren. Generell zeigt die Befragung aber auch, dass es für die Politik einige Ansatzpunkte gibt, bei denen sich Unternehmen eine gute Zusammenarbeit vorstellen können. Dazu zählen leistbares Wohnen sowie entsprechende Bildungsangebote vor Ort, zielgerichtete Förderschienen, die auf die lokalen Herausforderungen zugeschnitten sind, und nicht zuletzt das Framing ländlicher Regionen.

Dabei ist anzumerken, dass der Fokus der Befragung auf innovative Unternehmen gelegt wurde und somit deren Perspektive abgebildet wird. Personen aus Politik und Verwaltung wurden nicht befragt, weshalb keine Aussagen über die Einschätzung und Wahrnehmung der politischen Ebene getätigt werden können und die Ergebnisse nur unter diesen Rahmenbedingungen diskutiert werden sollten. 